\title{
Research on the Calculation Method of Rotation Axis
}

\author{
Xianyi Liu, Xihui Zhang, Zhaofa Zhou, Zhili Zhang, Zihao Xiu \\ Xi'an High Tech Istitute, Xi'an 710025 China \\ 785564025@qq.com
}

Keywords: Digital Zenith Camera, Optical Axis, Rotation Axis, Rotation Angle

\begin{abstract}
There exists axis errors when using the digital zenith camera to orient. And the misalignment between the optical axis and rotation axis will lead to much more error. In order to calculate the coordinate of the rotation axis, two star images in opposite direction are used. Then analyses the influence of the optical axis deviation and the rotation angle. The analysis of experimental data demonstrates that: the method is reasonable, the optical axis and the rotation axis are uncorrelated. When the rotation error is below 40 arc second, the influence of the rotation error can be ignored.
\end{abstract}

\section{Introduction}

The use of digital zenith camera for astronomical positioning is a high-precision astronomical positioning method[1,2]. The digital zenith camera shoots through the rotation of the star map in different orientations[3]. The star map is combined with the star chart for the identification of the star map and the image coordinate system between the image coordinate system and the celestial plane conversion relationship, After several iterations, the solution of the location of the station is realized[4].

In the positioning solution, the existence of the error will directly affect the accuracy of the solution. There are many sources of error in the process of positioning with digital zenith camera[5,6]. Which the existence of shaft error will bring a greater error. Because of the reason of the CCD installation, the optical axis and the axis of the digital zenith telescope will not coincide completely. Germany's Hirt uses the initial value iteration method to solve the value of the rotation axis[7]. Zeng Zhixiong proved that the astronomical coordinate of the optical axis of the symmetrical position can be used to calculate the astronomical coordinate value of the rotating axis in theory ${ }^{[8]}$. But the above method of solving the process are more complex. Zhang Hua-wei et al. Have also studied the problem of the solution of the rotating axis, but the research content is mainly embodied in theory and not concretely deduced with specific model ${ }^{[9]}$. For the rapid and effective solution of the coordinates of the rotation axis, in this paper, the coordinate of the rotation axis is directly solved by the symmetrical position using the four-parameter model, and the influence of the optical axis deviation and the rotation angle error on the rotation axis is analyzed theoretically.

\section{Astronomical Positioning Principle}

Digital zenith photographed the star map in the zenith, through the shooting of the star map to identify the establishment of image coordinates and celestial plane between the coordinates of the conversion relationship, the number of iterations after the final realization of the location of the location of the station. The CCD star sensor to capture the star point to identify, to establish the identification of the stars of the CCD image coordinates and celestial plane plane coordinates. Where the CCD image coordinates are based on the origin of the optical axis as the origin. The mapping relationship between image coordinates and celestial plane coordinates is established by four parameter transformation (Helmet transform). There are:

$$
\begin{aligned}
& u=-a x+b y+c_{1} \\
& v=b x+a y+c_{2}
\end{aligned}
$$


Where $(u, v)$ denotes the coordinates of the celestial plane of the star, and $(x, y)$ denotes the coordinates of the CCD image recognizing the star. $a b c_{1} c_{2}$ is the coordinate conversion parameter. Respectively, to resolve the symmetrical position of the image coordinates and the celestial plane between the coordinates of the conversion relationship. The initial position of the rotation axis is $(0,0)$. Respectively, to resolve the symmetry position of the satellite rotation axis of the celestial coordinates of $\left(\alpha_{1}, \delta_{1}\right)$ and $\left(\alpha_{2}, \delta_{2}\right)$, and take its average $(\alpha, \delta)$, as the rotation axis of the celestial coordinates.

$$
\begin{aligned}
& \alpha=\left(\alpha_{1}+\alpha_{2}\right) / 2 \\
& \delta=\left(\delta_{1}+\delta_{2}\right) / 2
\end{aligned}
$$

The solution of the coordinate axis of the rotating axis is transformed into the celestial tangent plane coordinates, The coordinates $\left(x_{1}, y_{1}\right)$ and $\left(x_{2}, y_{2}\right)$ of the axis of rotation of the axis of symmetry are calculated by (1), respectively, and the mean value $(x, y)$ is taken.

$$
\begin{aligned}
& x=\left(x_{1}+x_{2}\right) / 2 \\
& y=\left(y_{1}+y_{2}\right) / 2
\end{aligned}
$$

The newly settled value $(x, y)$ is used as the new image coordinate value of the rotation axis. Substituting the (1) formula to solve the rotation axis corresponding to the celestial plane plane coordinate value $\left(u_{z}, v_{z}\right)$, so as to solve the rotation axis corresponding to the new celestial coordinates $\left(\alpha_{z}, \delta_{z}\right)$.

$$
\begin{aligned}
& \alpha_{z}=\alpha_{0}+\arctan \frac{u_{z}}{\cos \delta_{0}-v_{z} \sin \delta_{0}} \\
& \delta_{z}=\arctan \frac{\left(v_{z}+\tan \delta_{0}\right) \cos \left(\alpha-\alpha_{0}\right)}{1-v_{z} \tan \delta_{0}}
\end{aligned}
$$

Repeat the above steps until the astronomical coordinate values that meet the accuracy requirements are obtained.

\section{A New Method Of Solving The Rotary Axis}

In the process of positioning, there is an inconsistency between the optical axis and the rotating shaft, that is, the optical axis and the rotation axis are not completely coincident with each other. Therefore, it is necessary to eliminate the error due to the inconsistency between the optical axis and the rotation axis. As shown in Fig. 1, the CCD causes the CCD to tilt due to mounting errors during the installation, so that the optical axis and the rotation axis are offset from each other. However, the angle between the rotary axis and the optical axis does not change during the rotation of the star image, and the optical axis rotates around the rotation axis.

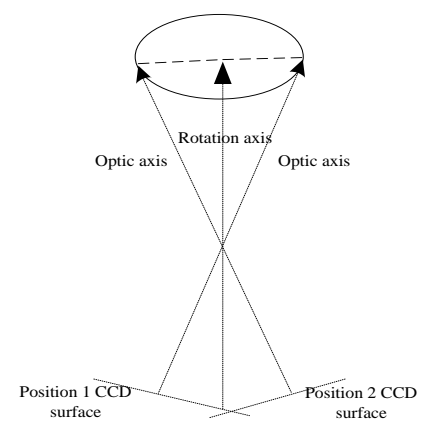

Fig. 1 The schematic diagram of axis

The value of the parameter $a b c_{1} c_{2}$ of the initial position and the value $a^{\prime} b^{\prime} c_{1}^{\prime} c_{2}^{\prime}$ of the symmetrical position parameter are respectively calculated using the least squares algorithm at the two positions of symmetry. As shown in Fig. 1, the image coordinates of the rotation axis in position 1 and position 2 are the same as those of the celestial plane, and the rotation axis is the common axis at 
position 1 and position 2, as shown in Fig. Let the rotation axis coordinate value of $\left(x_{r}, y_{r}\right)$, celestial plane plane coordinate value of $\left(u_{z}, v_{z}\right)$.

Set position 1 and position 2 to get:

$-\left(a-a^{\prime}\right) x_{r}+\left(b-b^{\prime}\right) y_{r}+\left(c_{1}-c_{1}^{\prime}\right)=0$

$\left(b-b^{\prime}\right) x_{r}+\left(a-a^{\prime}\right) y_{r}+\left(c_{2}-c_{2}^{\prime}\right)=0$

Order $m=a-a^{\prime}, \quad n=b-b^{\prime}, c=c_{1}-c_{1}{ }^{\prime}, \quad d=c_{2}-c_{2}{ }^{\prime}$

Solve the coordinates of the rotation axis

$$
\begin{aligned}
& x_{r}=(m c-n d) /\left(a^{2}+b^{2}\right) \\
& y_{r}=-(m d+n c) /\left(a^{2}+b^{2}\right)
\end{aligned}
$$

The new astronomical coordinates corresponding to the rotation axis are solved by rotating the axis image coordinatesRepeat the above calculations until the astronomical coordinates are met.

\section{The Effect of Optical Axis and Rotation Angle Error on the Axis Of Rotation}

In the positioning of the zenith, the use of the symmetric position of the star map to calculate the rotation axis, the rotation axis of the calculated value and the optical axis and rotation angle error has a direct relationship. Therefore, it is necessary to study the position of the rotating shaft from two aspects: the change of the position of the optical axis and the rotation angle error.

\subsection{The Effect of the Selection of the Optical Axis Position on the Position of the Rotary Axis}

In the actual use of digital zenith camera positioning process, the optical axis as the CCD center, the value of $(0,0)$. The image coordinate system is established with the optical axis as the coordinate origin, and the location of the station is realized by establishing the mapping relationship between the image coordinate system and the celestial plane coordinate system. However, in practice, some external factors such as optical distortion of the instrument result in some deviations in the center of the optical axis. This will cause changes in image coordinates. Assuming that the center axis deviation of the optical axis is $(\Delta x, \Delta y)$, the image coordinate system is established with the new optical axis center as the coordinate origin. The relationship between the new coordinate system and the original coordinate system is:

$$
\begin{aligned}
& x_{0}=x-\Delta x \\
& y_{0}=y-\Delta y
\end{aligned}
$$

Where $\left(x_{0}, y_{0}\right)$ is the coordinate value under the original coordinate. Then the original coordinate mapping can be expressed as:

$$
\begin{aligned}
& u=-a(x-\Delta x)+b(y-\Delta y)+c_{1} \\
& v=b(x-\Delta x)+a(y-\Delta y)+c_{2}
\end{aligned}
$$

Then $(x-\Delta x)$ and $(y-\Delta y)$ are regarded as a whole, you can solve the coordinates of the rotation axis is still $\left(x_{r}, y_{r}\right)$, which is

$$
\begin{aligned}
& x_{e}-\Delta x=x_{r} \\
& y_{e}-\Delta y=y_{r}
\end{aligned}
$$

Where $\left(x_{e}, y_{e}\right)$ the coordinate values of the rotation axes in the new coordinate system after the deviation of the optical axis values are obtained. Can be drawn

$$
\begin{aligned}
& x_{e}=\Delta x+x_{r} \\
& y_{e}=\Delta y+y_{r}
\end{aligned}
$$

The deviation value of the rotating shaft and the optical axis. There is no change in the position of the rotation axis on the CCD image. It is shown that the arbitrary selection of optical axis does not affect the calculation of the rotation axis. 


\subsection{The Effect of the Rotation Angle on the Position of the Rotary Axis}

Solving the position of the rotary axis requires a star map taken at a symmetrical position, but there is a corner error during the rotation. This causes the position of the rotary axis to change.

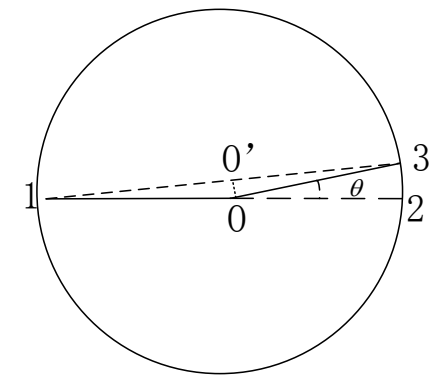

Fig. 2 The schematic diagram of rotation

As shown in Figure 2, position 1 is the initial position and position 2 is the ideal position after $180^{\circ}$ of rotation. Position 3 is the actual position after symmetrical rotation. In the ideal state, the rotary axis should be at the $\mathrm{O}$-point position, but due to the angular error during the rotation, the actual rotation angle does not reach the fully symmetrical state, and $\mathrm{O}$ 'is the actual coordinate of the rotation axis.

Due to the existence of the rotation angle error, the rotation axis has a certain deviation $\Delta \psi$

$$
\Delta \psi=r \cdot \sin \left(\frac{\theta}{2}\right)
$$

Where $r$ is the distance between the optical axis and the rotary axis, and $\theta$ is the rotation angle error value.

In the course of the experiment using the digital zenith camera pixel $4096 \times 4096$, the viewing angle of $3^{\circ} \times 3^{\circ}$, then we can see the CCD image resolution $A$

$$
A=\frac{F o v}{N}
$$

Where Fov is the field of view and $N$ represents the pixel value of a column. We can see the image resolution $A=2.6367 "$, in order to ensure that the rotation angle for the calculation of the results of the impact can be ignored. There is

$$
A \cdot r \cdot \sin \left(\frac{\theta}{2}\right) \leq 0.01^{\prime \prime}
$$

Can be solved $\theta \leq 40 "$ That is, when the rotation angle error is less than 40 ", the influence of the rotation angle error on the final settlement result is negligible.

\section{Experimental Data Processing and Analysis}

In the course of the experiment, a digital zenith camera was used to photograph the star map. A set of star images contained 16 star maps, and two star maps in the symmetrical position were used as a solution unit. The partial identification of the star data is given below.

Tab.1 Part of the recognized star data

\begin{tabular}{cccc}
\hline CCD x coordinate & CCD y coordinate & longitude & latitude \\
\hline 2004.696791 & 3772.154551 & 107.636131 & 33.430220 \\
3013.803313 & 3784.945373 & 107.030982 & 34.137424 \\
2615.436066 & 3576.527213 & 107.444305 & 33.958136 \\
1389.633333 & 3262.980952 & 108.425822 & 33.240049 \\
2816.903846 & 3670.307692 & 107.245322 & 34.054679 \\
3808.628866 & 566.680412 & 109.361091 & 36.259877 \\
320.031746 & 2334.666667 & 109.827796 & 32.917891 \\
623.065574 & 930.803279 & 110.869961 & 33.794310 \\
\hline
\end{tabular}

The CCD coordinate is the image coordinate system established by the starting point of CCD. It is transformed into the image coordinate system based on the center of the optical axis. Using the 
symmetrical position to directly solve the rotation axis position coordinates and the initial value iteration to solve the rotation axis position coordinates as shown in the following table:

Tab. 2 The rotation axis using two different methods

\begin{tabular}{cccc}
\hline \multicolumn{2}{c}{ Initial value iteration method } & \multicolumn{2}{c}{ Symmetric solution algorithm } \\
\hline X coordinate & Y coordinate & X coordinate & Y coordinate \\
41.02171 & 3.93586 & 41.02171 & 3.93586 \\
40.99302 & 3.59212 & 40.99302 & 3.59212 \\
41.23086 & 3.85760 & 41.23086 & 3.85760 \\
41.24952 & 4.11267 & 41.24952 & 4.11267 \\
40.89002 & 4.12508 & 40.89002 & 4.12508 \\
41.27124 & 4.58018 & 41.27124 & 4.58018 \\
41.19086 & 4.15827 & 41.19086 & 4.15827 \\
41.04287 & 4.13193 & 41.04287 & 4.13193 \\
\hline
\end{tabular}

It can be concluded that the coordinate value of the rotary axis calculated by the symmetrical position is consistent with the value calculated by the initial value iteration method, indicating the correctness of the symmetric solution algorithm.

In order to study the relationship between the coordinate of the rotation axis and the coordinate value of the optical axis, the coordinates of the optical axis are $(0,0)$, and the image coordinate system is established. And then the optical axis deviation value $(10,6)$, the establishment of the image coordinate system, the solution of the rotation axis in the new coordinates of the image coordinates.

Tab.3 The rotation axis since the optical axis changes

\begin{tabular}{cccc}
\hline \multicolumn{2}{c}{ Optical axis coordinate value $(0,0)$} & \multicolumn{2}{c}{ Optical axis deviation $(10,6)$} \\
\hline X coordinate & Y coordinate & X coordinate & Y coordinate \\
41.02171 & 3.93586 & 51.02171 & 9.93586 \\
40.99302 & 3.59212 & 50.99302 & 9.59212 \\
41.23086 & 3.85760 & 51.23086 & 9.85760 \\
41.24952 & 4.11267 & 51.24952 & 10.11267 \\
40.89002 & 4.12508 & 50.89002 & 10.12508 \\
41.27124 & 4.58018 & 51.27124 & 10.58018 \\
41.19086 & 4.15827 & 51.19086 & 10.15827 \\
41.04287 & 4.13193 & 51.04287 & 10.13193 \\
\hline
\end{tabular}

Table 3 shows that when the optical axis deviation, the value of the rotation axis will also produce the corresponding deviation value. However, relative to the entire CCD image, the position of the rotation axis does not change, but because the coordinates of the origin changes, the coordinate value in the new coordinate system under the corresponding changes only.

The astronomical coordinate values corresponding to the position of the optical axis before and after the optical axis change and the astronomical coordinate values corresponding to the rotation axes are calculated.

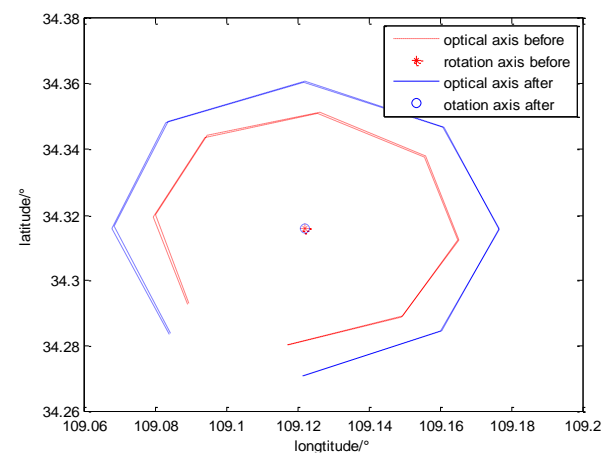

Fig.3 The astronomy coordinates since the optical axis changes

It can be seen from the figure that the change of the coordinate value of the optical axis will cause the astronomical coordinates of the optical axis to change, but the celestial coordinates of the rotating 
axis remain unchanged, that is, the astronomical coordinates of the rotating axis do not change with the coordinate value of the optical axis The This also indicates that the change in the optical axis does not change the position of the rotary axis.

\section{Conclusion}

Using the symmetrical position to directly solve the rotation axis position coordinates simplifies the process of solving, and the value of the solution is consistent with the value calculated by the initial value iteration. The coordinate value of the rotary axis is affected by the rotation angle error, but when the error value of the rotation angle is within, the influence of the rotation angle error on the final settlement result is negligible. In addition, the value of the rotation axis will produce the corresponding deviation value with the deviation of the optical axis. However, the position of the rotary axis on the CCD does not change, indicating that the astronomical coordinate value of the rotary axis does not correspond to the position of the optical axis Changes have changed.

\section{References}

[1]. Wang Bo,Tian Li-li,Wang Zheng,et al.The image and data processing in digital zenith camera camera [J]. Chin.Sci.Bull,2014,59(12):1100-1107.

[2]. Zhai Guang-qin,Ai Gui-bin.Digital zenith camera camera astronomical positioning measurement of project implementatiion[J].Journal of Geomatics Science and Technology, 2014, 31(3): 232-235.

[3]. Lili Tian,Jinyun Guo,Yanben Han.et al.Digital zenith camera telescope prototype of China[J].Chin.Sci.Bull,2014,59(17):1978-1983.

[4]. Christian Hirt,Automatic determination of vertical deflections in real-time by combining GPS and digital zenith camera camera for solving the GPS-height-problem[C].Proc.14th Int.Technical Meeting of the Satellite Division of the Institude of Navigation.2001,2540-2551.

[5]. Guo Jin-yun,Song Lai-yong,Chang Xiao-tao, et al.Vertical deflection measure with digital zenith camera camera and accuracy analysis[J]. Geomatics and Information Science of Wuhan University,2011,36(9):1085-1088.

[6]. R.Schoebel, G.W.Hein, B.Eissfeller.Renaissance of Astrogeodetic Levelling Using GPS/CCD Zenith Camera [J].Schriftenreihe Studiengang Vermessungswesen, 2004, 63(5):45-52.

[7]. Christian Hirt,Beat Burki,Anna Somieski,et al. Modern Determination of Vertical Deflections Using Digital zenith camera Camera[J].Journal ofSurveying Engineering.2010,9(2):1-12.

[8]. Zheng Zhi-xiong,Hu Xiao-dong,Gao Li-min.Effect of digital zenith camera camera axis error on measurement precision of vertical deflection and its correction[J].Optics and Precision Engineering,2004,12(1):6-10.

[9]. Zhang Hua-wei,Ai Gui-bin,Wei Dai-yong.et al.The method to solve the rotate axis in digital zenith camera camera[P].China:2011110406263.9,2012.06.27. 